Document downloaded from:

http://hdl.handle.net/10251/98274

This paper must be cited as:

Ortuño-Lizarán, I.; Vilariño, G.; Martínez-Ramos, C.; Monleón Pradas, M.; Vallés Lluch, A. (2016). Influence of synthesis parameters on hyaluronic acid hydrogels intended as nerve conduits. Biofabrication. 8(4):1-12. doi:10.1088/1758-5090/8/4/045011

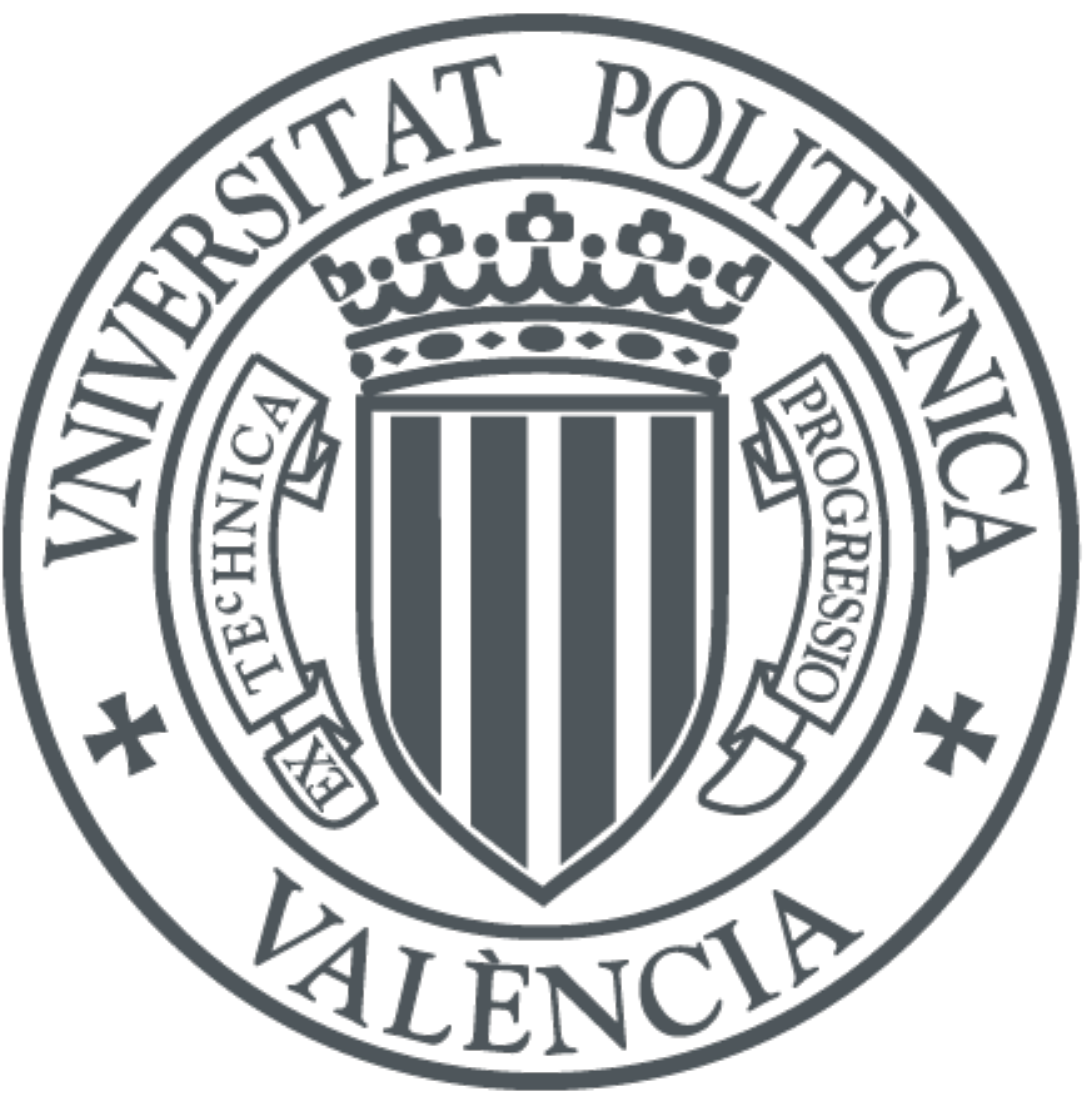

The final publication is available at

https://doi.org/10.1088/1758-5090/8/4/045011

Copyright IOP Publishing

Additional Information 


\section{Influence of synthesis parameters on hyaluronic acid hydrogels intended as nerve conduits}

Isabel Ortuño-Lizarán, ${ }^{\dagger, \ddagger, \# ~ G u i l l e r m o ~ V i l a r i n ̃ o-F e l t r e r, ~}{ }^{\dagger, \#}$ Cristina Martínez-Ramos, ${ }^{\dagger, \#}$ Manuel Monleón Pradas, ${ }^{\dagger, \ddagger, \# ~ a n d ~ A n a ~ V a l l e ́ s-L l u c h ~}{ }^{\dagger, \#, *}$

${ }^{\dagger}$ Centre for Biomaterials and Tissue Engineering, Universitat Politècnica de València, Cno. de Vera s/n, 46022, Valencia, Spain $†$

${ }^{\ddagger}$ Networking Research Center on Bioengineering, Biomaterials and Nanomedicine (CIBER-BBN), Spain

* Corresponding author. E-mail address: avalles@ter.upv.es

\# All authors contributed equally to this work 


\section{Abstract}

Hydrogels have widely been proposed lately as strategies for neural tissue regeneration, but there are still some issues to be solved before their efficient use in tissue engineering of trauma, stroke or the idiopathic degeneration of the nervous system. In a previous work of the authors a novel Schwann-cell structure with the shape of a hollow cylinder was obtained using a threedimensional conduit based in crosslinked hyaluronic acid as template. This original engineered tissue of tightly joined Schwann cells obtained in a conduit lumen having $400 \mu \mathrm{m}$ in diameter is a consequence of specific cell-material interactions. In the present work we analyze the influence of the hydrogel concentration and of the drying process on the physicochemical and biological performance of the resulting tubular scaffolds, and prove that the cylinder-like cell sheath obtains also in scaffolds of a larger inner diameter. The diffusion of glucose and of the protein BSA through the scaffolds is studied and characterized, as well as the enzymatic degradation kinetics of the lyophilized conduits. This can be modulated from a couple of weeks to several months by varying the concentration of hyaluronic acid in the starting solution. These findings allow to improve the performance of hyaluronan intended for neural conduits, and open the way to scaffolds with tunable degradation rate adapted to the site and severity of the injury.

Keywords: hyaluronan; scaffold; nerve conduit; Schwann cell; degradability 


\section{Introduction}

The treatments of soft tissue damages and disorders have traditionally focused on lessening their related symptoms through pharmacotherapy, surgery or physiotherapy in an attempt to improve patients' quality of life [1-3]. More recently efforts have been directed towards regeneration of such damaged tissues; herein, tissue engineering plays a key role, especially in areas such as the central nervous system regeneration, which seemed unapproachable until amazing findings in the last three decades [4-7] proved that neurogenesis existed in the adult brain. Some current approaches in nervous system tissue engineering are based on the graft of stem or differentiated cells $[4,8-10]$, sometimes supported with bioactive molecules. For instance, mature Schwann cells have been broadly used already for neurological treatments, inasmuch they mediate myelination of peripheral nervous system axons and contribute to regeneration after injury by differentiating and proliferating. Moreover, they produce neurotrophic factors (like BDNF or NGF), cell adhesion molecules (NCAM, integrins, L1CAM) and extracellular matrix proteins (laminin or collagen) which could theoretically support axonal growth by creating a milieu propitious to regeneration [11].

However, a number of studies showed that most cells grafted this way in a host lack a tridimensional context able to guide axonal regrowth in order to regenerate neuronal pathways. For this reason, cell therapy assisted by three-dimensional scaffolds, which can act as drug carriers as well, has been widely studied [12] in order to maximize the chance of cell survival and growth. Specifically, for nervous system tissue engineering, the use of guidance tubular structures (commonly named as nerve conduits) is one of the more promising approaches [13]. Theoretically, a tubular structure could act as an axonal bridge between two ends of a damaged nerve or neural tract, allowing proliferation of glial cells and axonal regeneration within its lumen $[14,15]$. Therefore, an ideal material for this purpose should have a channeled and porous structure in order to be permeable to nutrients, neurotrophic factors or other signaling molecules of the environment. Nevertheless, the conduit wall should be impermeable to cells, to isolate the grafted cells from the feasible host immune response in order to support their growth along the material. Besides, biodegradable scaffolds are preferred, as they would progressively disappear, 
being substituted by new extracellular matrix while the tissue is being regenerated $[13,16]$. Biopolymers like polysaccharides and other extracellular matrix components are among the few candidate materials that can lead into constructs gathering all the properties of an ideal nerve conduit $[17,18]$.

Hyaluronic acid (HA) is an interesting material because of its inherent cell-friendly properties. HA is a natural glycosaminoglycan, major component of the extracellular matrix, composed of repeating units of $\beta-1-4-\mathrm{D}$-glucuronic acid and $\beta-1-3-\mathrm{N}$-acetyl-D-glucosamine [19-21]. This polymer has many advantages for its use in tissue engineering, as it is biocompatible, biodegradable, useful as a drug release system, and its degradation products have been described as angiogenic and beneficial for cellular proliferation [22-24]. Several problems concerning the use of HA hydrogels for tissue engineering can be overcome by chemically crosslinking the polymer chains in order to improve their mechanical properties and handling of the material $[25,26]$ without compromising their biological performance. Through these chemical modifications, HA hydrogels can be cast in molds to form stable and insoluble nerve conduits. Moreover, post-processing techniques like lyophilization contribute to obtain porous substrates for cells to proliferate and interact with [27-29].

In this work, a novel tubular porous biomaterial based on crosslinked HA combined with SCs in its lumen is proposed as guidance conduit for regeneration of nervous pathways. The main objective of this work is to critically compare and assess the physicochemical and biological effect of two key factors during HA scaffolds manufacture: stock HA-DVS formulations and post-processing drying. This study extends previous findings [30] where threelayered porous HA conduits with $400 \mu$ m-lumen diameter, prepared starting from a fixed HA concentration in the solution and lyophilized were presented. Here we address the effect the initial HA concentration and of the drying procedure on the porous structure of the conduits, the diffusion of small and large molecules through the resulting HA network, and its degradation. Further we prove that the engineered Schwann cell structure reported previously obtains also when the diameter is more than doubled. 


\section{Materials and methods}

\subsection{Preparation of hyaluronic acid conduits}

The synthesis of neural conduits of crosslinked HA with a three-layered wall porosity was carried out based on the procedure previously described [30], with the following variations affecting HA concentration and lyophilization. Here, 3 and 5\% wt/wt hyaluronic acid (from Streptococcus equi, 1,5-1,8 MDa, Sigma-Aldrich) solutions in 0.2M sodium hydroxide ( $\mathrm{NaOH}$; Scharlau) were prepared by gently mixing for $24 \mathrm{~h}$. Then, the HA was crosslinked with divinyl sulfone (Sigma-Aldrich), as described by Balazs [31], at a 90\% molar ratio (9 DVS moles per 10 moles of HA monomeric units), after stirring for 10 seconds, and pouring the mixture in a grooved mould.

The mould, made of polytetrafluoroethylene (PTFE) and with $2 \mathrm{~mm}$ squared-section grooves, was intended for obtaining the tubular conduits, using a $1 \mathrm{~mm}$-diameter poli- $\varepsilon$-caprolactone (PCL; Polysciences) fiber as template of the inner channel (400 $\mu \mathrm{m}$ in 30). 2 mm-diameter PTFE washers were set in the ends and the center of the PCL fiber.

Solutions were allowed to gel for 30 (5\% HA) or $60 \mathrm{~min}(3 \% \mathrm{HA})$ and subsequentely frozen at $-20^{\circ} \mathrm{C}$ for a minimum of $5 \mathrm{~h}$. From that point, conduits were produced under two different conditions: lyophilization and conventional drying (hereafter termed non-lyophilized samples). The first set was lyophilized (LyoQuest 85 , Telstar Life Science Solutions) for $24 \mathrm{~h}$ at $-80^{\circ} \mathrm{C}$ and $20 \mathrm{~Pa}$, while the second set was air-dried at room temperature for $15 \mathrm{~h}$. Then, the PCL fibers were removed from the conduits, and there were swollen in distilled water for $2 \mathrm{~h}$. Finally, the PCL fibers were extracted and the conduits were washed in a 50:50 acetone:water mixture for the first $48 \mathrm{~h}$ and with distilled water for 3 days, changing the rinsing solution every $24 \mathrm{~h}$ in order to remove unreacted DVS and $\mathrm{NaOH}$. Four different scaffold types were thus obtained, according to the HA starting concentration and the drying method, labeled as HA3L (3\% lyophilized HA), HA5L (5\% lyophilized HA), HA3nL (3\% non-lyophilized HA), and HA5nL (5\% non-lyophilized HA). 


\subsection{Preparation of hyaluronic acid discs}

Following the procedure described above, lyophilized 3, 3.5, 4, 4.5 and 5\% HA membranes and air-dried (non-lyophilized) 3 and 5\% membranes of 1-2 mm thickness were obtained by casting the reactant HA solutions onto Petri dishes and allowing to gel for between 2 (for 3\% membranes) or $1 \mathrm{~h}$ (for $5 \%$ membranes) at $37^{\circ} \mathrm{C}$. The membranes were die-cut into small discs of $5 \mathrm{~mm}$ diameter. The discs were labelled 3L, 3.5L, 4L, 4.5L, 5L (lyophilized) and 3nL, 5nL (air-dried) to differentiate samples from conduits.

\subsection{Morphological characterization of HA conduits}

Lyophilized and air-dried conduits were observed by scanning electron microscopy (SEM, Hitachi S-4800, Hitachi High-Technologies Canada Inc., Toronto, Canada). Materials were gold-coated and pictures of longitudinal and transversal sections of them were taken at a $10 \mathrm{~mm}$ working distance and $10 \mathrm{kV}$.

\subsection{Density and porosity measurements}

Density and porosity of the HA3L, HA5L, HA3nL and HA5nL conduits $(\mathrm{N}=4)$ were gravimetrically determined with the density kit of a precision balance (AE-240, Mettler Toledo, Greifensee, Switzerland). The conduits were weighted both dry (mass $m_{d}$ ) and immersed in $n-$ octane (Sigma-Aldrich, density $\rho_{o}=0.703 \mathrm{~g} / \mathrm{cm}^{3}$ ) (mass $\mathrm{m}_{\mathrm{im}}$ ), and Archimedes' principle was used as in equation 1 , where $\rho_{H A}$ is the density of the material. The scaffolds' pores were previously filled with n-octane under vacuum for 5 min to ensure that the solvent filled their pores.

$$
\rho_{H A}=\frac{m_{d}}{m_{\text {im }}} \rho_{o}
$$

The pore volume fraction, $\pi$, was determined with equation 2 , by weighting samples when their pores were filled with n-octane but not immersed in the solvent, $m_{\mathrm{d} o}$.

$$
\pi=\frac{V_{\text {pores }}}{V_{\text {total }}}=\frac{m_{d o}-m_{d}}{m_{d o}-m_{\text {im }}}
$$




\subsection{Degradation and stability assays}

An enzymatic degradation assay on crosslinked HA was performed with 3 and 5\% air-dried and 3, 3.5, 4, 4.5 and 5\% lyophilized discs in order to systematically analyze both the effect of the different drying procedures and the concentration of HA in the solution. The assay was carried out immersing the samples $(\mathrm{N}=4)$ in a solution of bovine testis hyaluronidase type IV-S (BTH, Sigma-Aldrich) $10 \mathrm{U} / \mathrm{mL}$ in phosphate buffer saline, DPBS, at $37^{\circ} \mathrm{C}$. Stability of the discs in a non-enzymatic aqueous solution was assessed by immersing another series of samples in the same volume of DPBS at $37^{\circ} \mathrm{C}$. The enzyme was thermally inactivated $\left(1.5 \mathrm{~min}\right.$ at $\left.100^{\circ} \mathrm{C}\right)$ at withdrawal at different degradation times (from 0 to 81 days). Next, the discs were vacuumdried to remove the buffer, and analyzed as to their morphology with SEM (after 0, 16 and 81 days of degradation), and to their mass loss during the experiments. Fractional mass loss was calculated using equation 3 , where $m_{0}$ and $m_{t}$ are the initial weight and the weight at time $t$, respectively.

$$
\text { fractional mass loss }=\frac{\mathrm{m}_{0}-\mathrm{m}_{\mathrm{t}}}{\mathrm{m}_{0}}
$$

\subsection{Diffusion assays}

In order to evaluate the wall permeability of the conduits produced, the diffusion of bovine serum albumin (BSA, Roche) and glucose (Sigma-Aldrich), taken here as neural model molecules for metabolites and nutrients, respectively, was assessed. Four different conduit types (HA3L, HA5L, HA3nL and HA5nL) were used in this experiment, using 4 replicates of each one. One end of the conduits was sealed with a 5\% HA via-DVS crosslinking solution. Once gelled, the conduits' lumen was filled with $7 \mu \mathrm{l}$ of a concentrated BSA (30 mg/ml) or glucose (100 $\mathrm{mg} / \mathrm{ml})$ solution and the other end was sealed likewise. Materials were then placed in a multiwell plate, whose wells were filled with $1 \mathrm{ml}$ of deionized water, and the plate was sealed and incubated at $37^{\circ} \mathrm{C}$. Aliquots of half the volume of the well were taken at different times, and the volume withdrawn was replaced with fresh deionized water. Concentrations of BSA and glucose aliquots were determined by means of colorimetric assays, using the BCA protein assay 
kit (23235, Thermo Scientific, Barcelona, Spain) and glucose oxidase reaction [32], respectively. Briefly, $1 \mathrm{~mL}$ of BCA mix was added to $1 \mathrm{~mL}$ of the sample and incubated $1 \mathrm{~h}$ at $60^{\circ} \mathrm{C}$. Then, absorbance was read at $562 \mathrm{~nm}$ in a Victor Multilabel Counter 1420 (Perkin Elmer, Waltham MA; USA) For the glucose determination, a Trinder solution $(0.3 \mathrm{U} / \mathrm{mL}$ horseradish peroxidase, $8.5 \mathrm{mM}$ phenol and $0.3 \mathrm{mM}$ 4-aminoantipiryne, all from Sigma-Aldrich) with 10 U/mL glucose oxidase (Sigma-Aldrich, Madrid, Spain) was prepared. It was then mixed in a 1:5 vol. proportion with the sample and, after 5 min of reaction at room temperature, absorbance was measured at $505 \mathrm{~nm}$. According to the established procedure, BSA or glucose released cumulative mass at each time $n$ was calculated following equation $4, m_{a}$ being the aliquot mass.

$$
\text { released } \operatorname{mass}_{0 \rightarrow n}=2 \cdot m_{a_{-} n}+\sum_{i=1}^{n-1} m_{a_{-} i}
$$

The released cumulative mass data were fitted to a diffusion model. The model is that of a long hollow cylinder of inner and outer radius $r_{a}$ and $r_{b}$, respectively, with an initial concentration of solute inside, immersed in a solution and with the following initial and boundary conditions:

$$
\begin{gathered}
C(r, 0)=C_{0} \quad r \leq r_{b} \\
C(r, t)=C_{1} \approx 0 \quad t \geq 0 ; r \geq r_{b}
\end{gathered}
$$

where $C_{0}$ is the initial solute (glucose or BSA) concentration and $C_{1}$ refers to the medium. Fu et al[33] proposed equation 5 to describe the fickian diffusion of such a problem, considering similar diffusive properties for both the cylinder wall and the liquid inside it.

$$
\frac{M_{t}}{M_{\infty}}(t)=1-\frac{4}{r_{b}^{2}} \sum_{n}^{\infty} \frac{\exp \left[-D \cdot \alpha_{n}^{2} t\right]}{\alpha_{n}^{2}}
$$

Herein, $M_{t}$ is the weight difference relative to the starting value, $M_{\infty}$ is the weight difference attained at very long times, $D$ is the Fick's diffusion coefficient and $\alpha_{n}$ is the $n^{\text {th }}$ positive root satisfying $J_{0}\left(r_{b} \alpha_{n}\right)=0, J_{0}$ being the zero-order Bessel function of first kind. Mathcad software (PTC, Needham, USA) was used to calculate both the $D$ parameter for the best fit with the $5^{\text {th }}$ first $\alpha_{n}$ roots and the standard error of such data regression, $s_{\text {reg }}$. 


\subsection{Sanitization of the materials}

HA3L, HA5L, HA3nL and HA5nL conduits were immersed in $70^{\circ}, 50^{\circ}$ and $30^{\circ}$ ethanol solutions for $2 \mathrm{~h}, 10 \mathrm{~min}$ and $10 \mathrm{~min}$, respectively. Afterwards, 3 rinses of $10 \mathrm{~min}$ with type- 1 water were done to remove the ethanol, and the conduits were left overnight with culture medium at $37^{\circ} \mathrm{C}$ before starting the biological assays.

\subsection{Cell seeding on conduits}

Rat schwann cells (SCs, Innoprot, Bizkaia, Spain) at their $5^{\text {th }}$ passage were used in this experiment. The cells in the flask were washed with PBS and then detached with trypsin/EDTA (Life Technologies, Barcelona, Spain). Trypsin was blocked with Dulbecco’s Modified Eagle Medium (DMEM high glucose 4.5 g/L, Life Technologies, Barcelona, Spain) containing 10\% fetal bovine serum (FBS, Life Technologies, Barcelona, Spain). The medium with the cells was collected and centrifuged at $1200 \mathrm{rpm}$ for $5 \mathrm{~min}$. Then, the pellet was suspended in Schwanncell medium (Innoprot, Bizkaia, Spain) and quantified using a Neubauer chamber. SCs were seeded in the lumen of HA3L, HA3nL, HA5L and HA5nL conduits $\left(5 \times 10^{4}\right.$ cells per each $6 \mathrm{~mm}$ long conduit), placed in a 48 -well plate and incubated for $30 \mathrm{~min}$ at $37^{\circ} \mathrm{C}$ and $5 \% \mathrm{CO}_{2}$. Fresh Schwann-cell medium was next added to each well. The culture environment was maintained at $37^{\circ} \mathrm{C}$ and $5 \% \mathrm{CO}_{2}$ for 14 days, changing the medium every $48 \mathrm{~h}$.

\subsection{Cell viability and proliferation}

The (4,5-dimethylthiazol-2-yl)-5-(3-carboxymethoxyphenyl)-2-(4-sulfophenyl)-2H-tetrazolium salt viability assay (MTS, Cell titer 96 Aqueous One Solution cell proliferation assay, Promega, USA) was performed after 1,7 and 14 days of cell culture, to evaluate cell behavior within different conduits. 4 replicates per time were used. Shortly, cultured conduits were rinsed three times in PBS and next a DMEM without phenol red (Life Technologies, Barcelona, Spain):MTS 5:1 solution was added to each well. Samples were incubated protected from light for $3 \mathrm{~h}$ at $37^{\circ} \mathrm{C}$ and $5 \% \mathrm{CO}_{2}$. The plate containing the samples was shaken the first and last $5 \mathrm{~min}$ of the incubation time to ensure the MTS solution and its product were homogenously distributed 
inside the lumen. Then, two aliquots per well were placed in a new 96-well plate and absorbance at $490 \mathrm{~nm}$ was read. Since the gap between cells' behavior when cultured in 2D and 3D platforms has been widely studied already, making them both incomparable, the use of coverslips as control was intentionally avoided.

Live and dead cell rate was evaluated over culture time using a LIVE/DEAD assay kit (Life Technologies, Barcelona, Spain). Cultured conduits were rinsed three times in PBS for 5 min each and the dye solution (Ethidium homodimer $4 \mu \mathrm{M}$ and calcein in DPBS $2 \mu \mathrm{M}$ ) was added and incubated for $45 \mathrm{~min}$ at $37^{\circ} \mathrm{C}$, in absence of light. Samples were observed under an epifluorescence microscope (Nikon Eclipse 80i).

\subsection{Cell morphology analysis}

Immunocytochemistry was performed after 14 culture days. Cells were rinsed three times with PBS, fixed with 4\% paraformaldehyde (PFA, Panreac, Spain) for 20 min at room temperature, and next washed twice with PBS at $4^{\circ} \mathrm{C}$. Cell membranes were permeabilized with a blocking buffer (BB), based in DPBS + 1\% BSA (Sigma-Aldrich, Madrid, Spain) $+0.1 \%$ Tween20 (Sigma-Aldrich, Madrid, Spain) for 1 hour. Then, a primary antibody (mouse anti-GFAP, 1/300 dilution in BB, Abcam) was added to each conduit and incubated overnight at $4^{\circ} \mathrm{C}$. Next, samples were rinsed twice with DPBS and incubated for $2 \mathrm{~h}$ with a secondary antibody (goat anti-mouse Alexa 647, 1/200 dilution, Jackson Immunoresearch) and BODIPY FL Phallacidin (1/40 dilution, Life Technologies, Barcelona, Spain). Finally, the nuclei were stained for $10 \mathrm{~min}$ with 4',6-diamidino-2-phenylindole dihydrochloride (DAPI, 1/5000 dilution in PBS, SigmaAldrich, Madrid, Spain). Conduits were cut longitudinally and fluorescence images were taken in a CLSM 780 confocal microscope (Carl Zeiss AG, Oberkochen, Germany).

Besides, SEM images were obtained after 14 days of culture in order to observe the cell morphology and arrangement along the scaffolds' lumen. Conduits were rinsed three times with PBS. Then they were fixed with 3.5\% glutaraldehyde (GA, Electron Microscopy Science, Spain) for $1 \mathrm{~h}$ at $37^{\circ} \mathrm{C}$, washed twice with $\mathrm{PBS}$, post-fixed with $2 \%$ osmium tetroxide $\left(\mathrm{OsO}_{4}\right.$, Aname, Spain) for $1.5 \mathrm{~h}$ and rinsed four times with cold distilled water, 10 min each. To 
condition them for SEM, samples were dehydrated with sequential incubations in graded cold ethanol solutions (30/50/70/96/100, 10 min each). Then the ethanol was exchanged for liquid carbon dioxide and samples were critical point-dried and coated. Transversal and longitudinal images of the channel were obtained under SEM at $10 \mathrm{kV}$ and $10 \mathrm{~mm}$ of working distance.

\subsection{Statistical analysis}

All experiments in this study were performed at least three times (unless otherwise specified) and the data are presented as mean \pm standard deviation (SD). For the density, porosity and MTS assay data, an ANOVA test together with a multiple sample mean comparison (Tuckey's HSD test with a significance degree of 95\%) was performed to reveal significant differences between conditions. When significant differences were found between sample variances $\left(\mathrm{s}^{2}\right)$, paired Student's t-tests were used instead of Tuckey's HSD test. All tests were performed with the Statgraphics Centurion XVI statistics software (Statpoint Technologies, Warrenton, USA). 


\section{Results and discussion}

\subsection{Surface characterization of hyaluronic acid conduits}

The fabrication method described in the present work allowed to obtain $6 \mathrm{~mm}$-long, squarecross section scaffolds of $2 \mathrm{~mm}$ side, with a $1 \mathrm{~mm}$-diameter inner cylindrical channel. Morphology and porosity as seen by SEM is given in Figure 1. The longitudinal channel edges are highlighted with a dashed white line in images A to D. The air-dried conduits showed a smooth surface without pores, while the lyophilized group had a distribution of interconnected honeycomb-like pores of around $60 \mu \mathrm{m}$ in diameter. HA5L conduits had a more regular and homogeneous structure than HA3L ones, which, in addition, were considerably softer when handling them. As for the lumen surface of lyophilized conduits (images $\mathrm{C}$ and D) the porosity is lower and with smaller pores ( 2 to $15 \mu \mathrm{m}$ ) than inside the walls ( $\mathrm{E}$ and $\mathrm{F}$ ). When hydrated, however, only the lyophilized conduits kept their integrity and similar dimensions. The hydrated non-lyophilized conduits swelled more water, compared to its dry condition, and they were considerably more sensitive to pressure tearing.

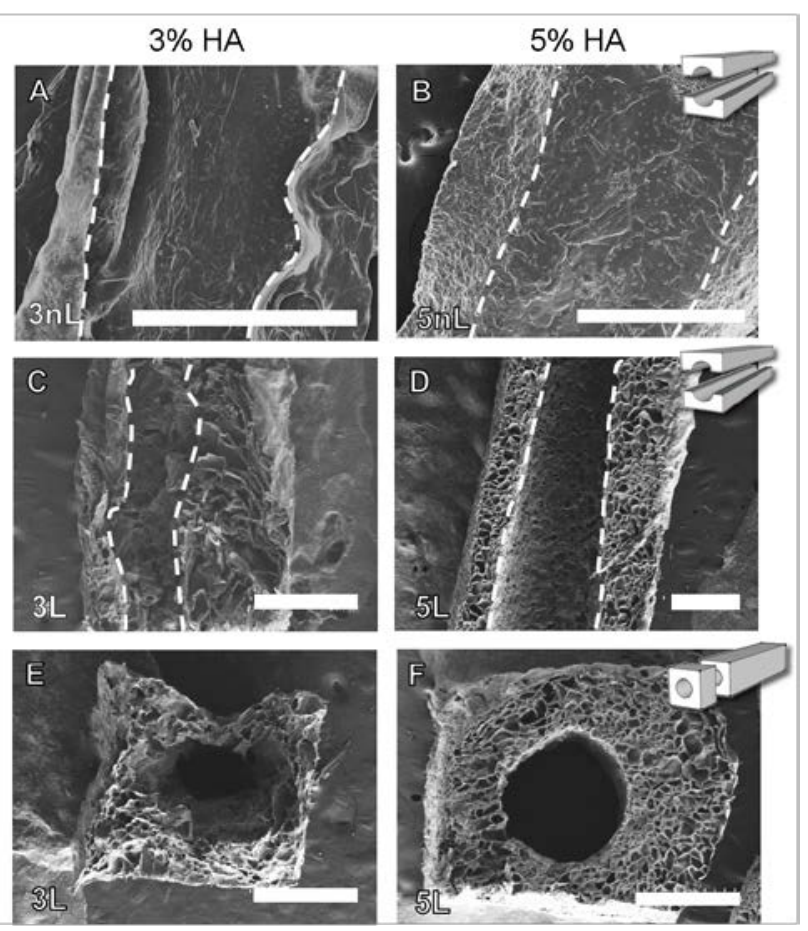

Figure 1. SEM images of longitudinal (A-D) and transversal (E-F) sections of non-lyophilized (A-B) and lyophilized (C-F) HA conduits. Insets show porosity details of each image. Scale bar: $800 \mu \mathrm{m}$ (A-B) and $1 \mathrm{~mm}$ (C-F). 


\subsection{Density and porosity of the conduits' HA matrix}

The mean density values of the crosslinked HA polymer networks ranged between 1.17 and $1.42 \mathrm{~g} / \mathrm{cm}^{3}$, with no significant differences between the groups of study (Figure 2A), although a slight increase may be discerned for the high HA concentration groups. As for the conduits' porosity (Figure 2B), the drying procedure clearly influenced the pore volume fraction: all lyophilized conduits presented porosities above 83\% whilst air-dried samples had porosities around $50 \%$ or smaller. 

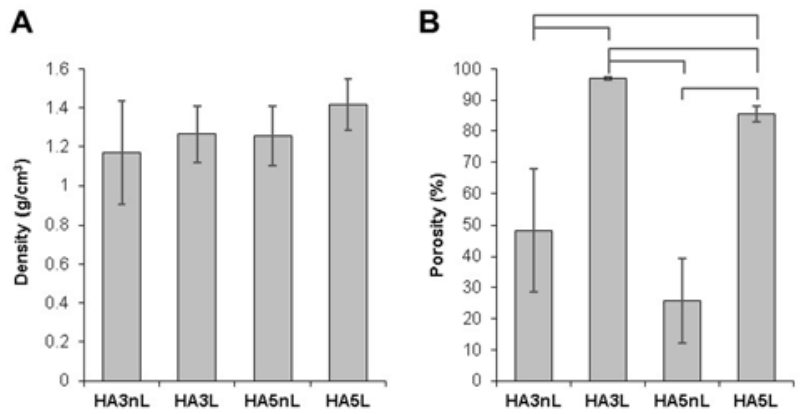

Figure 2. Density (A) and porosity (B) of the HA3nL, HA3L, HA5nL and HA5L. No statistical significant differences were found between groups in A. Statistically significant differences ( $\mathrm{p}<$ 0.05) in mean values between pairs in $\mathbf{B}$ are represented by horizontal bars.

The air-dried conduits had a defined lumen when hydrated, but their walls were more irregular and lost thickness. The lyophilized samples, by contrast, kept in the dry state a honeycomb pore structure across their thickness. Freeze-drying can lead to this particular structure in crosslinked HA, which in other cases has been obtained with porogenic techniques such as gas foaming, or injection in porogen templates.[34,35] However, the unusual relation between water molecules and hyaluronic acid chains substructure and stiffness leads to changes in molecular conformation during the lyophilization that confers the conduits unique properties, as showed in Figures 1, 2 and subsequent assays [36]. The porosities of the non-lyophilized HA3nL and HA5nL samples were thus significantly lower.

\subsection{Degradation and stability of conduits}

Macroscopically, a faster degradation rate in 3L discs was observed (Figure 3), compared with 5L ones. Figure 3A shows the size decrease of 3L samples relative to the control materials (incubated without enzyme), which kept their size constant. Controls were thus not photographed at all degradation times (in the image, they are placed below the corresponding enzymatically degraded sample). Contrarily, the size of 5L discs remained apparently stable over time in enzymatic medium. This fact was further confirmed microscopically by SEM 
images, Figure 3B to 3F, after 16 and 81 days of degradation. Figure $\mathbf{3 C}$ reveals an almost complete degradation of 3L samples after 16 days, when they were reduced to a few particles (encircled by a dashed line on the Figure). 5L discs, however, kept their structural integrity after 3 months. They showed an increase in pores number and size over degradation time (images D to F), though, probably due to the mass loss following bulk hydrolysis. Because of this significant difference between lyophilized samples prepared from different starting concentrations, it was decided to follow the mass loss of samples prepared from intermediate concentrations as well.
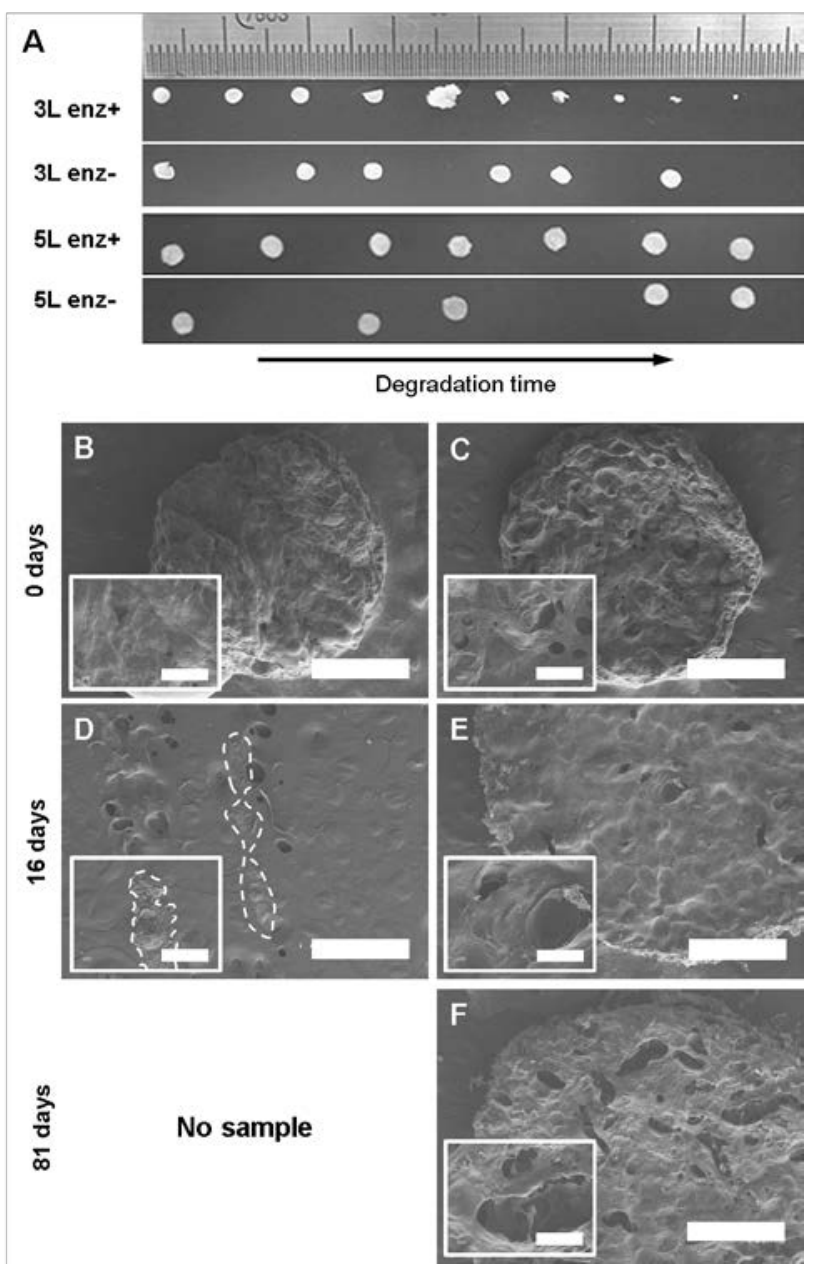

Figure 3. A) Evolution of 3L and 5L over the degradation time in enzymatic (enz+, top line) and non-enzymatic (enz-, bottom line) medium, respectively. Graded scale in centimeters. B-F) SEM images of the surface of 3L (B-C) and 5L (D-F) discs at different degradation times. 
Insets show porosity details of each image. The dashed line in $\mathbf{C}$ outlines HA residues reduced to powder. Scale bar: $1 \mathrm{~mm}$; details: $200 \mu \mathrm{m}$.

Mass loss was systematically quantified over degradation time for conduits prepared with starting solutions of HA concentration between 3 and 5\%, Figure 4. These results confirm the degradation trend inferred from Figure 3: 3L samples were almost entirely degraded in about 16 days, while 5L discs maintained their mass nearly constant ( $<20 \%$ mass loss in 81 days). Furthermore, a concentration-dependent effect was found for lyophilized samples, though not a direct function of the concentration, but greater for concentrations above $4 \%$. As for the differences in the degradation rate with the drying procedure, the $3 \mathrm{~nL}$ samples remained far more stable in terms of mass loss than the 3L lyophilized ones, whilst no significant differences were found when the HA concentration increased to $5 \%$.
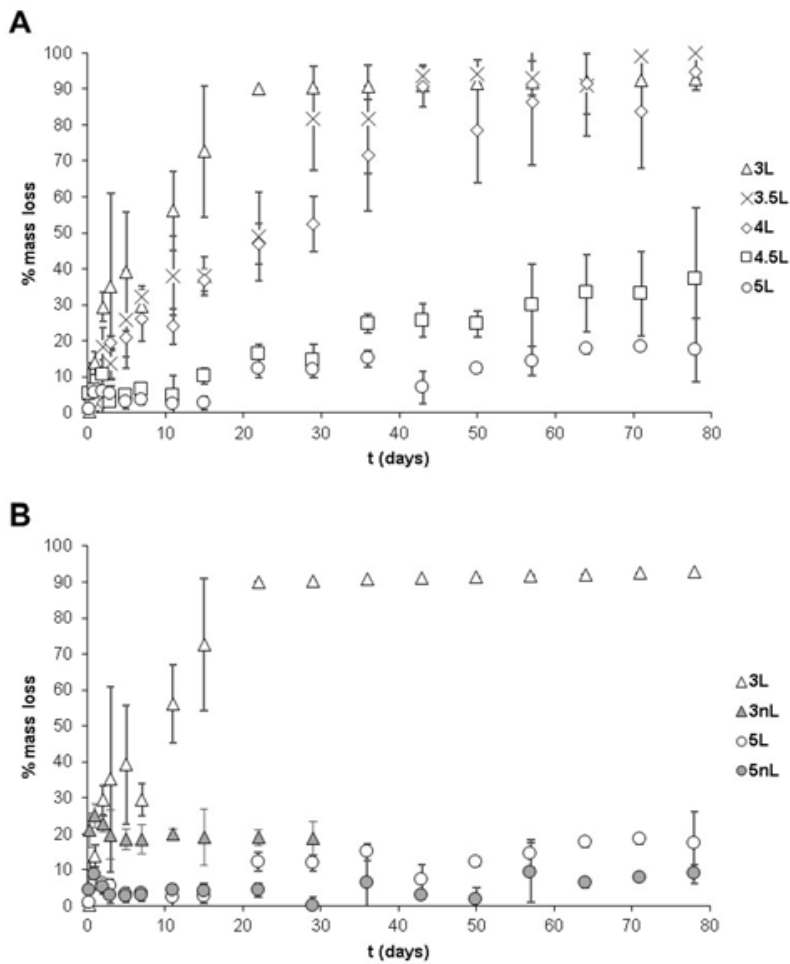

Figure 4. Fractional mass loss percentage of 3L, 3.5L, 4L, 4.5L and 5L discs (A) and 3L, 3nL, 5L and $5 \mathrm{~nL}$ discs (B) over time. No data were collected beyond 28 days for $3 \mathrm{~nL}$ samples, for which no significant differences were detected in the last 6 mean values shown. 
Regardless of DVS-crosslinking, the hyaluronidase enzymatic potential remained intact, probably because the $[1 \rightarrow 4]$ glycosidic bonds are not transformed during the HA-DVS reaction. Since only the conduits which undergo lyophilization were remarkably affected by the enzyme over time, the most feasible explanation is that their highly porous structure permitted a higher bulk diffusion of the hyaluronidase to degrade the scaffold from the inside, whilst the more compact homogeneous structure of the air-dried conduits prevented the same bulk-type degradation. As for the lyophilized HA substrates (3L, 3.5L, 4L, 4.5L and 5L), their degradation rate is clearly HA concentration-dependent, probably due to the increase in structure homogeneity and honeycomb-like pores' wall thickness as the HA initial concentration increases, as it can be inferred from Figures 1C to 1F. Moreover, conduits made up of HA at 3\% $\mathrm{wt} / \mathrm{wt}$ and lyophilized swelled considerably more immersed in pure water or buffer media than HA5L ones (see reference[30]), implying more glycosidic bonds accessible to water molecules and, therefore, to the dissolved enzyme. This may lead to structural failures which fracture the 3D scaffold into fragments, easier to be degraded by the enzyme into simple oligosaccharides. Even so, it looks like the enzymatic degradation has a critical point between 4 and $4.5 \%$ HA above which the hyaluronidase efficiency is somewhat lower and the conduits remain mechanically stable for longer. The modulation of the effective degradation time from a couple of weeks to several months just by modifying a simple synthesis factor (the HA concentration in the solution) represents a remarkable result, considering that scaffolds for regenerative medicine applications should degrade over the course of tissue regeneration to allow complete restoration by host tissue [37-39].

\subsection{Diffusion of molecules through the conduits wall}

Diffusion experiments revealed a different release profile depending on whether the conduits had been previously lyophilized or air-dried, and such differences were also found between conduits of different HA initial concentration (3 or 5\%). 


\begin{tabular}{|c|c|c|}
\hline Conduit & $\begin{array}{c}D_{B S A} \times 10^{10} \\
{\left[\mathrm{~m}^{2} / \mathrm{s}\right]}\end{array}$ & $\begin{array}{c}D_{\text {glucose }} \times 1_{10}{ }^{10} \\
{\left[\mathrm{~m}^{2} / \mathrm{s}\right]}\end{array}$ \\
\hline HA3nL & $8.2(0.054)$ & $4.06(0.063)$ \\
\hline HA3L & $2.87(0.031)$ & $4.50(0.054)$ \\
\hline HA5nL & $4.95(0.029)$ & $7.93(0.045)$ \\
\hline HA5L & $2.75(0.047)$ & $4.46(0.059)$ \\
\hline
\end{tabular}

Table 1. Diffusion coefficients for BSA and glucose through the different conduits' wall swollen in water. The number in brackets corresponds to $s_{\text {reg }} /$ range $\equiv s_{\text {reg }}$ of each condition, since, by definition, $M_{t} / M_{\infty}$ range is $[0,1]$.

Figure 5 shows the percentage of the initial loaded mass that is released as a function of time (notice that the quantity represented as ordinate of these plots is not the left-hand side of equation (5)). The release kinetics for both BSA and glucose through the conduits' wall, as characterized by their diffusion coefficients, is similar, with $D$ around $5 \times 10^{-10} \mathrm{~m}^{2} / \mathrm{s}$, as listed in Table 1. However, the tubes release a much smaller fraction of BSA (between $24 \%$ and $35 \%$ for HA3nL and HA5nL, respectively) than of glucose (Figure 5A).
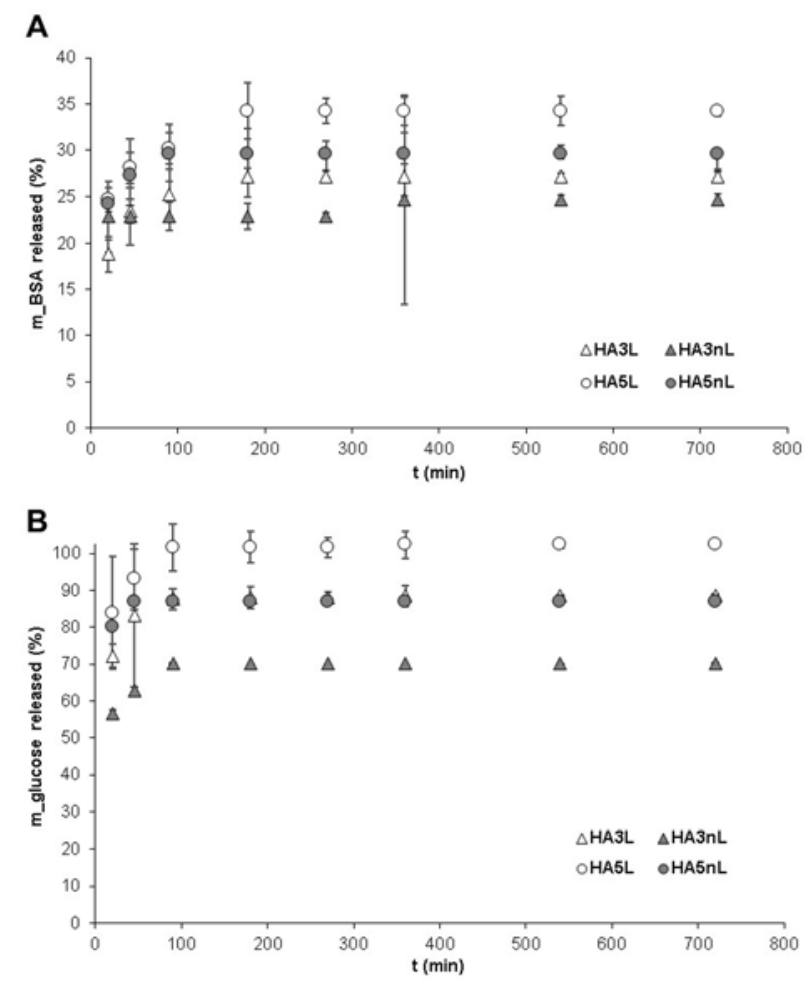

Figure 5. Percentage of BSA (A) and glucose (B) initial mass released through the wall of HA3L, HA3nL, HA5L and HA5nL conduits over time. 
These low values of equilibrium BSA release may be due to protein-HA chain interactions, since HA interacts with several extracellular matrix proteins [40,41] and BSA is a large molecule with many interaction sites. Since lyophilization and higher HA concentration in the preparation procedure lead to a higher macroporosity and homogeneity of the conduit's wall, HA5L conduits were those that allowed the maximum release of glucose and BSA.

\subsection{Cell viability and proliferation}

Absorbance of aliquots is directly proportional to the number of metabolically active cells, thus it can be said that the viability assays on SCs cultures (Figure 6) revealed a higher cell survival and proliferation on HA5L conduits. There are little differences between HA3nL, HA5nL and HA5L conduits at day 1 of culture, but at longer culture times absorbance decreases in HA3nL and HA5nL conduits, suggesting cell death or detachment. In HA3L conduits SCs kept a constant population density. In HA5L conduits, absorbance increased steadily along the experiment, reaching a mean value more than three times higher than after the first $24 \mathrm{~h}$.

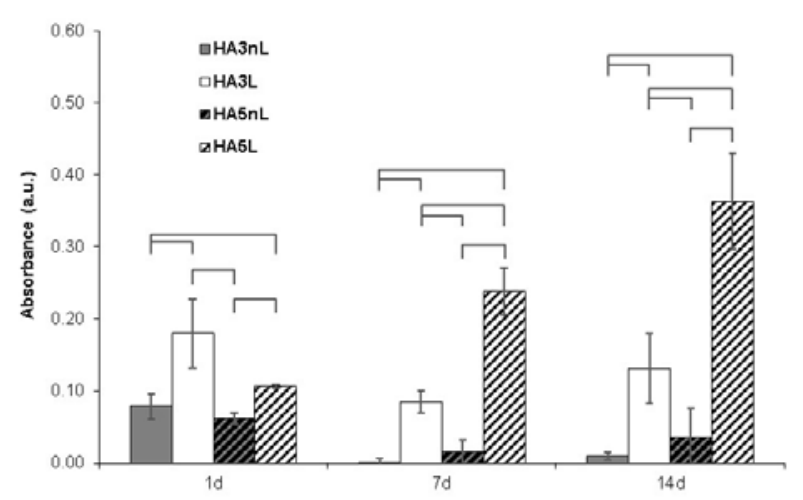

Figure 6. MTS viability assay data for HA3nL, HA3L, HA5nL and HA5L conduits with SCs cultured in their lumen for 1,7 and 14 days. Statistically significant differences $(p>0.05)$ between pairs for each time point are represented by horizontal bars.

The LIVE/DEAD assay (Figure 7) confirmed such results. Only a very small number of spherically-shaped cells were found in the lumen of HA3nL and HA5nL conduits after 14 days, 
stained in red. In the HA3L and HA5L groups, most of the cells showed green staining and were uniformly spread on the lumen surface, completely covering it in case of the HA5L conduits (Figure 7D) and largely in HA3L ones (Figure 7B).
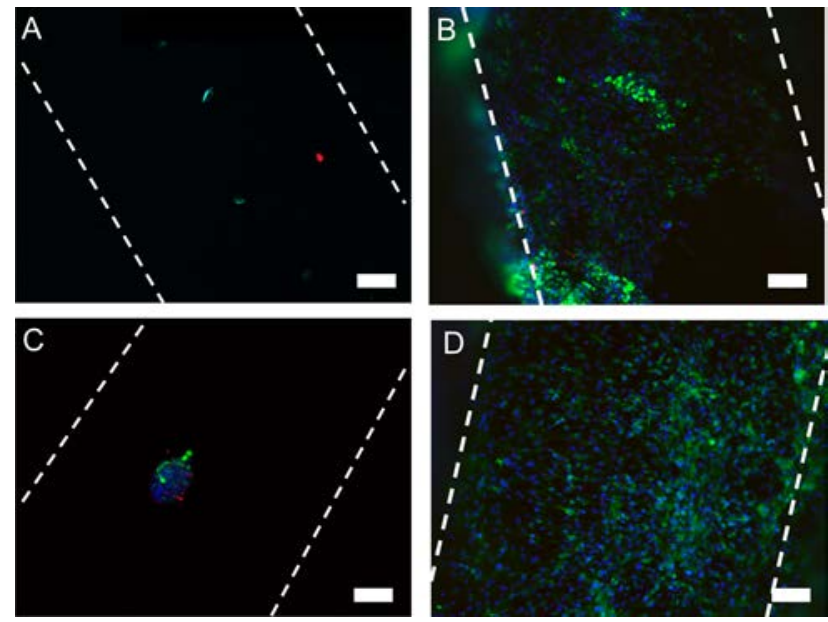

Figure 7. Fluorescence images of the LIVE/DEAD assay after a 14 days culture in HA3nL (A), HA3L (B), HA5nL (C) and HA5L (D) conduits, stained with DAPI (blue), calcein (green) and ethidium homodimer staining (red). Images show the longitudinal cuts of the conduits. The dashed lines outline the edges of the lumen. Scale bar: $100 \mu \mathrm{m}$.

\subsection{Morphological characterization of cells cultured in conduits}

Immunocytochemistry for glial marker GFAP (red) of cells cultured inside HA5L conduits assessed the SCs characteristic phenotype under normal metabolic conditions (Figure 8). Phallacidin stain (green) revealed F-actin, a main component of the cytoskeleton, that revealed a predominant pattern for the orientation of the cells' soma (Figure 8B, C) along the conduits' channel main direction. 

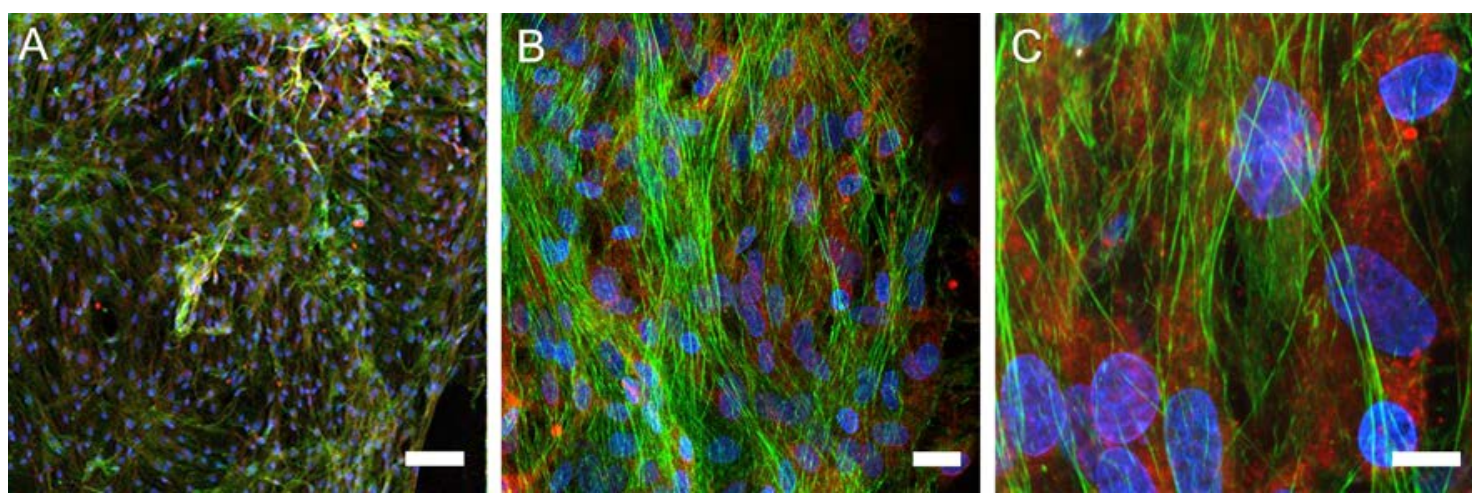

Figure 8. Confocal microscopy images of Schwann-cell seeded HA5L conduits at their $14^{\text {th }}$ culture day immunostained with GFAP (red), Phallacidin (green) and DAPI (blue). B and C show details of A. Scale bar: $100 \mu \mathrm{m}$ (A), $20 \mu \mathrm{m}$ (B), $10 \mu \mathrm{m}$ (C).

The cell arrangement in the lumen of the HA5L conduits can be well seen on SEM images (Figure 9). SCs grew constrained in the interior of the channel (since no cells were observed inside the conduit's wall), and completely coated the lumen's surface. Cells seemed to form a dense but thin sheath through tight interactions. The cell-sheath was around $20 \mu \mathrm{m}$ thick, the dimensions of a monolayer composed of $2-3$ stacked cells.
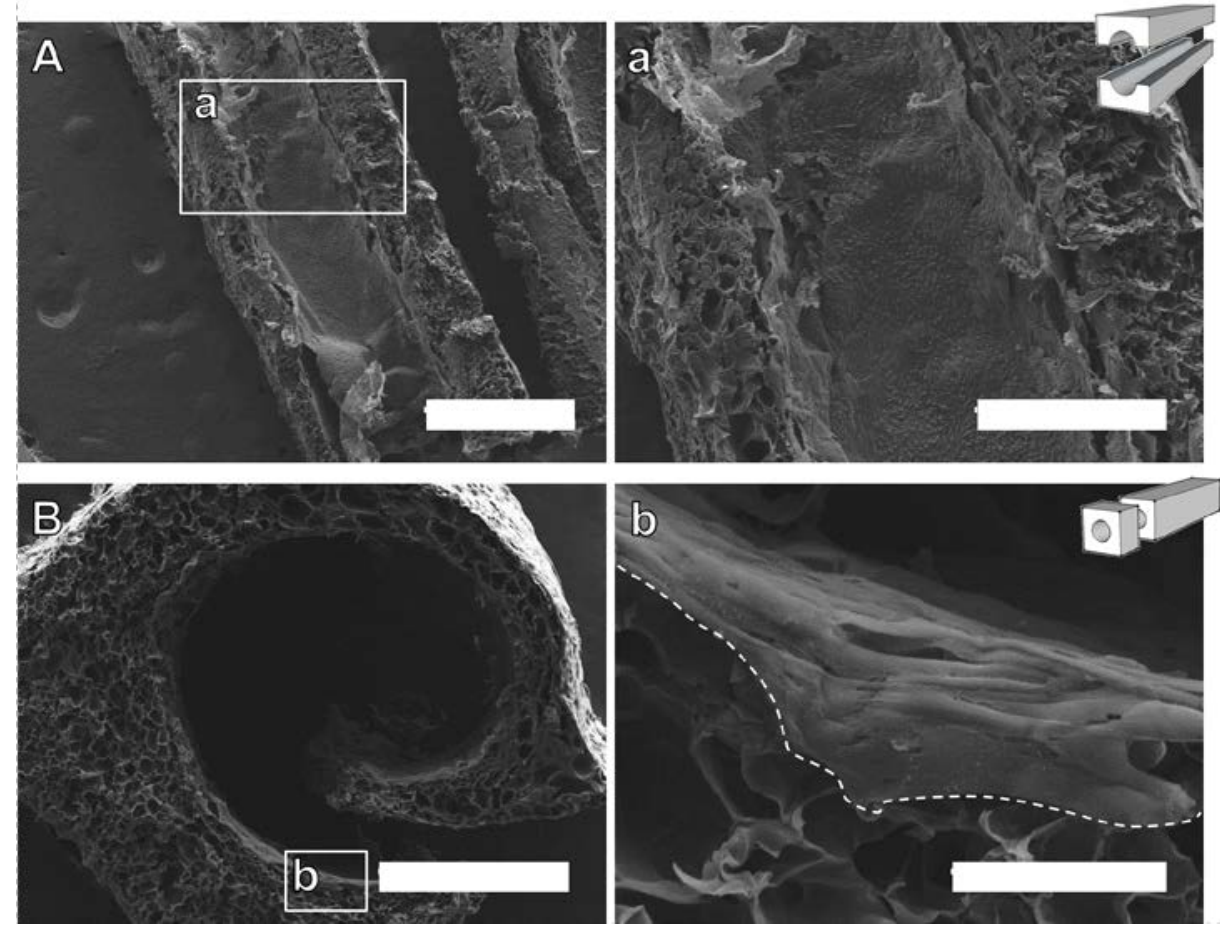
Figure 9. SEM images of longitudinal (A) and transversal sections (B) of SCs inside a HA5L conduit after 14 culture days. Images on the right are magnifications of the rectangle highlighted on the left images. The dotted line in $\mathbf{b}$ distinguishes the cells coating from the conduit wall. Scale bar: 1 mm (A), $500 \mu \mathrm{m}$ (a, B), $50 \mu \mathrm{m}$ (b).

These results reveal that Schwann cells are able to spread and grow within the HA conduits' internal channel for 14 days, regardless of the well-known HA low adherent properties [42-44], but only in the lyophilized conduits HA3L and HA5L. In all cases, SCs remained confined inside the conduits' lumen during the whole culture span, but in the air-dried conduits the flatter surface was not a friendly environment to attach and proliferate. In contrast, the abundant pores in the lyophilized scaffolds represented a 3D substrate where to adhere and nourish through. The pore size on the surface of the conduits' lumen is small enough to impede SCs migration through the porous wall, but permissible for fluid and nutrient flows guaranteeing cell viability inside the conduits. Thus, these conduits may act as a vehicle for protected cell transplantation in the organism.

In the culture conditions within the HA5L conduits SCs could proliferate to the extent of making a dense thin SC sheath, uniformly coating the lumen's surface, composed of multiple SCs tightly joined together. The issue of the lumen diameter is critical and must be stressed at this point, since both the radius of curvature of the surface and the proper weight of the number of cells forming the cylinder sheet represent important challenges. The results here reported prove that the singular cell structure which was previously obtained in conduits of $400 \mu \mathrm{m}$-inner diameter $^{30}$ can also be generated when the diameter of the scaffold's channel is more than doubled.

The methodology herein presented can consequently be used to create biohybrids containing a sheath of longitudinally aligned SCs, where other cell types (such as neural precursors), drugs and growth factors $[45,46]$ can further be incorporated, to finally conform an implantable construct for neural regeneration. 


\section{Conclusions}

Synthesis parameters of HA conduits were studied for their commitment on the biological performance of the conduits with SCs, in view of their use as neural conduits. The concentration of HA in the reactive mixture and the post-gelation drying procedure (air-drying vs. lyophilization) were analyzed. Their effect on the enzymatic degradation rate, swelling behavior and molecule permeability across the conduits' wall were assessed, and cultures of Schwann cells within the conduits were studied for cell viability, proliferation, phenotype and morphology.

Lyophilization leads to easier-to-handle highly porous structures, through which nutrients and waste products could flow easier than in tighter air-dried ones. The initial HA concentration can be used to tailor swelling and degradation rate. The HA5L formulation resulted in conduits with well-connected honeycomb-like pores, with trabeculae able to withstand the structure collapse during drying. Such a stable porous structure allowed diffusion of molecules but impeded seeded cells to migrate through the conduit's wall. Further it supplied the seeded cells with adhesion sites required for proliferation. Thus, Schwann cells could colonize completely the lumen to eventually form a continuous thin sheath of tightly joined cells.

\section{Author information}

Corresponding Author *E-mail: avalles@ter.upv.es. Tel: +34 961877007, ext. 88936 Fax: +34 963877276.

\section{Acknowledgments}

The authors acknowledge Spanish Ministerio de Ciencia e Innovación through projects PRIPIMNEU-2011-1372 (ERANET-Neuron), MAT2011-28791-C03-02 and -03. I. Ortuño Lizarán acknowledges support by CIBER-BBN starting grant. 


\section{References}

[1] Devos D, Moreau C, Dujardin K, Cabantchik I, Defebvre L and Bordet R 2013 New pharmacological options for treating advanced parkinson's disease Clin. Ther. 35 164052

[2] Speed C a 2001 Therapeutic ultrasound in soft tissue lesions. Rheumatology (Oxford). 40 $1331-6$

[3] Jibuike O O, Paul-Taylor G, Maulvi S, Richmond P and Fairclough J 2003 Management of soft tissue knee injuries in an accident and emergency department: the effect of the introduction of a physiotherapy practitioner. Emerg. Med. J. 20 37-9

[4] Berry M 1986 Neurogenesis and gliogenesis in the human brain Food Chem Toxicol 24 79-89

[5] Eriksson P S, Perfilieva E, Bjork-Eriksson T, Alborn A M, Nordborg C, Peterson D A and Gage F H 1998 Neurogenesis in the adult human hippocampus Nat Med 4 1313-7

[6] Murrell W, Bushell G R, Livesey J, McGrath J, MacDonald K P, Bates P R and MackaySim A 1996 Neurogenesis in adult human Neuroreport 7 1189-94

[7] Alvarez-Buylla A and García-Verdugo J M 2002 Neurogenesis in adult subventricular zone J. Neurosci. 22 629-34

[8] Braak H and Del Tredici K 2008 Assessing fetal nerve cell grafts in Parkinson's disease. Nat. Med. 14 483-5

[9] Tennstaedt A, Aswendt M, Adamczak J, Collienne U, Selt M, Schneider G, Henn N, Schaefer C, Lagouge M, Wiedermann D, Kloppenburg P and Hoehn M 2015 Human neural stem cell intracerebral grafts show spontaneous early neuronal differentiation after several weeks Biomaterials 44 143-54

[10] Papastefanaki F, Chen J, Lavdas A A, Thomaidou D, Schachner M and Matsas R 2007 Grafts of Schwann cells engineered to express PSA-NCAM promote functional recovery after spinal cord injury Brain 130 2159-74

[11] Fortun J, Hill C E and Bunge M B 2009 Combinatorial strategies with Schwann cell transplantation to improve repair of the injured spinal cord Neurosci Lett 456 124-32

[12] Ricks C B, Shin S S, Becker C and Grandhi R 2014 Extracellular matrices, artificial neural scaffolds and the promise of neural regeneration Neural Regen Res 9 1573-7

[13] Schmidt C E and Leach J B 2003 Neural tissue engineering: strategies for repair and regeneration Annu Rev Biomed Eng 5 293-347

[14] Olson H E, Rooney G E, Gross L, Nesbitt J J, Galvin K E, Knight A, Chen B, Yaszemski M J and Windebank A J 2009 Neural stem cell- and Schwann cell-loaded biodegradable polymer scaffolds support axonal regeneration in the transected spinal cord. Tissue Eng. Part A 15 1797-805

[15] Sinis N, Schaller H-E, Schulte-Eversum C, Schlosshauer B, Doser M, Dietz K, Rösner H, Müller H-W and Haerle M 2005 Nerve regeneration across a 2-cm gap in the rat median nerve using a resorbable nerve conduit filled with Schwann cells. J. Neurosurg. 103 1067-76

[16] Hudson T W, Evans G R and Schmidt C E 2000 Engineering strategies for peripheral nerve repair Orthop Clin North Am 31 485-98

[17] Cullen D K, Tang-Schomer M D, Struzyna L a., Patel A R, Johnson V E, Wolf J a. and Smith D H 2012 Microtissue Engineered Constructs with Living Axons for Targeted Nervous System Reconstruction Tissue Eng. Part A 18120817094501006

[18] Jansen K, Van Der Werff J F A, Van Wachem P B, Nicolai J P A, De Leij L F M H and Van Luyn M J A 2004 A hyaluronan-based nerve guide: In vitro cytotoxicity, 
subcutaneous tissue reactions, and degradation in the rat Biomaterials 25 483-9

[19] Lam J, Truong N F and Segura T 2014 Design of cell-matrix interactions in hyaluronic acid hydrogel scaffolds Acta Biomater 10 1571-80

[20] Lei Y, Gojgini S, Lam J and Segura T 2011 The spreading, migration and proliferation of mouse mesenchymal stem cells cultured inside hyaluronic acid hydrogels Biomaterials 32 39-47

[21] Lepperdinger G, Fehrer C and Reitinger S 2004 Chemistry and Biology of Hyaluronan

[22] Day A J and De La Motte C A 2005 Hyaluronan cross-linking: A protective mechanism in inflammation? Trends Immunol. 26 637-43

[23] Milner C M, Higman V A and Day A J 2006 TSG-6: a pluripotent inflammatory mediator? Biochem. Soc. Trans. 34 446-50

[24] West D C, Hampson I N, Arnold F and Kumar S 1985 Angiogenesis induced by degradation products of hyaluronic acid. Science 228 1324-6

[25] Hallen L, Johansson C and Laurent C 1999 Cross-linked hyaluronan (Hylan B gel): a new injectable remedy for treatment of vocal fold insufficiency--an animal study Acta Otolaryngol 119 107-11

[26] Collins M N and Birkinshaw C 2007 Comparison of the effectiveness of four different crosslinking agents with hyaluronic acid hydrogel films for tissue-culture applications $J$. Appl. Polym. Sci. 104 3183-91

[27] Ibrahim S, Kang Q K and Ramamurthi A 2010 The impact of hyaluronic acid oligomer content on physical, mechanical, and biologic properties of divinyl sulfone-crosslinked hyaluronic acid hydrogels J. Biomed. Mater. Res. - Part A 94 355-70

[28] Rnjak-Kovacina J, Wray L S, Burke K a, Torregrosa T, Golinski J M, Huang W and Kaplan D L 2015 Lyophilized Silk Sponges: A Versatile Biomaterial Platform for Soft Tissue Engineering. ACS Biomater. Sci. Eng. 1 260-70

[29] Yu C, Bianco J, Brown C, Fuetterer L, Watkins J F, Samani A and Flynn L E 2013 Porous decellularized adipose tissue foams for soft tissue regeneration Biomaterials $\mathbf{3 4}$ 3290-302

[30] Vilariño-Feltrer G, Martínez-Ramos C, Monleón-De-La-Fuente A, Vallés-Lluch A, Moratal D, Barcia Albacar J A and Monleón Pradas M 2016 Schwann-cell cylinders grown inside hyaluronic-acid tubular scaffolds with gradient porosity Acta Biomater. $\mathbf{3 0}$ 199-211

[31] Balazs, E A and Leshchiner A 1986 Cross-linked gels of hyaluronic acid and products containing such gels Med Devices 4 1-10

[32] Trinder P 1969 Determination of blood glucose using an oxidase-peroxidase system with a non-carcinogenic chromogen J Clin Pathol 22 158-61

[33] Fu J C, Hagemeir C and Moyer D L 1976 A unified mathematical model for diffusion from drug-polymer composite tablets. J. Biomed. Mater. Res. 10 743-58

[34] Kim J K, Kim H J, Chung J Y, Lee J H, Young S B and Kim Y H 2014 Natural and synthetic biomaterials for controlled drug delivery Arch Pharm Res 37 60-8

[35] Annabi N, Nichol J W, Zhong X, Ji C, Koshy S, Khademhosseini A and Dehghani F 2010 Controlling the porosity and microarchitecture of hydrogels for tissue engineering Tissue Eng Part B Rev 16 371-83

[36] Gribbon P, Heng B C and Hardingham T E 2000 The analysis of intermolecular interactions in concentrated hyaluronan solutions suggest no evidence for chain-chain association. Biochem. J. 350 Pt 1 329-35

[37] Hosseinkhani M, Mehrabani D, Karimfar M H, Bakhtiyari S, Manafi A and Shirazi R 
2014 Tissue engineered scaffolds in regenerative medicine World J Plast Surg 3 3-7

[38] Bitar K N and Zakhem E 2014 Design strategies of biodegradable scaffolds for tissue regeneration Biomed Eng Comput Biol 6 13-20

[39] Asti A and Gioglio L 2014 Natural and synthetic biodegradable polymers: different scaffolds for cell expansion and tissue formation Int J Artif Organs 37 187-205

[40] Ojha B and Das G 2011 Role of hydrophobic and polar interactions for BSA-amphiphile composites Chem. Phys. Lipids 164 144-50

[41] Martins M, Azoia N G, Shimanovich U, Matam?? T, Gomes A C, Silva C and CavacoPaulo A 2014 Design of novel BSA/hyaluronic acid nanodispersions for transdermal pharma purposes Mol. Pharm. 11 1479-88

[42] Chen S H, Chen C H, Shalumon K T and Chen J P 2014 Preparation and characterization of antiadhesion barrier film from hyaluronic acid-grafted electrospun poly(caprolactone) nanofibrous membranes for prevention of flexor tendon postoperative peritendinous adhesion Int $J$ Nanomedicine 9 4079-92

[43] Smit X, van Neck J W, Afoke A and Hovius S E 2004 Reduction of neural adhesions by biodegradable autocrosslinked hyaluronic acid gel after injury of peripheral nerves: an experimental study $J$ Neurosurg 101 648-52

[44] Erturk S, Yuceyar S, Temiz M, Ekci B, Sakoglu N, Balci H, Dirican A, Cengiz A and Saner H 2003 Effects of hyaluronic acid-carboxymethylcellulose antiadhesion barrier on ischemic colonic anastomosis: an experimental study Dis Colon Rectum 46 529-34

[45] Godinho M J, Teh L, Pollett M A, Goodman D, Hodgetts S I, Sweetman I, Walters M, Verhaagen J, Plant G W and Harvey A R 2013 Immunohistochemical, Ultrastructural and Functional Analysis of Axonal Regeneration through Peripheral Nerve Grafts Containing Schwann Cells Expressing BDNF, CNTF or NT3 PLoS One 8

[46] Nie X, Deng M, Yang M, Liu L, Zhang Y and Wen X 2014 Axonal Regeneration and Remyelination Evaluation of Chitosan/Gelatin-Based Nerve Guide Combined with Transforming Growth Factor-??1 and Schwann Cells Cell Biochem. Biophys. 68 163-72 